\title{
Effectiveness of a Brief Multi- Component Intervention to HIV Prevention Among Spanish Youth
}

\begin{tabular}{|l|}
\hline Rafael Ballester-Arnal, $1 \square$ \\
Phone: +34 964729726 \\
Email: rballest@uji.es \\
\hline María Dolores Gil-Llario, 2 \\
\hline Estefanía Ruiz-Palomino, 1 \\
\hline Cristina Giménez-García, 1 \\
\hline
\end{tabular}

${ }^{1}$ Department of Clinical and Basic Psychology and Psychobiology, Universitat Jaume I of Castellón, Avda. Vicent Sos Baynat s/n, 12071 Castellón de la Plana, Spain

2 Department of Developmental and Educational Psychology, Universitat de València, Av. Blasco Ibáñez, 21, 46010 Valencia, Spain

\section{Abstract}

The greater rate of HIV infection occurs before age 30 in Spain. Our aim was to evaluate the long-term impact of a brief HIV preventive intervention on key sexual risk behavior components in Spanish young population. Participants were 467 young people aged 18-25 years who participated in B-PAPY intervention. They were evaluated at four times: a week, a month, three months and one year after intervention. A self-administered instrument was used. It was found a significant increase from pre-test to follow-ups in HIV knowledge, HIV susceptibility perception, confidence in condom and use of condoms. There were decreases statistically significant in the perceived severity of AIDS. The HIV intervention showed effectiveness over time in the main risk variables for HIV infection. Brief multi-component HIV interventions would be a useful resource to generalize HIV prevention.

\section{Resumen}


En España, la mayor prevalencia de infección por VIH ocurre antes de los 30 años. Nuestro objetivo ha sido evaluar el impacto a largo plazo de un programa breve de prevención del VIH en jóvenes españoles, que incluía los componentes clave del comportamiento sexual de riesgo. La muestra estaba formada por 467 jóvenes de 18 a 25 años, que participaron en la intervención B-PAPY. Se realizaron 4 seguimientos: una semana, un mes, tres meses y un año después de la intervención. Se utilizó un instrumento autoadministrado. Los resultados mostraron un aumento significativo del conocimiento, la vulnerabilidad percibida, la confianza en el condón y la conducta preventiva. Se encontraron cambios estadísticamente significativos en la gravedad atribuida al Sida. La intervención se mostró eficaz en las principales variables de riesgo para la infección por VIH. Las intervenciones multicomponentes breves pueden ser un recurso útil para la generalización de la prevención del VIH.

\section{Keywords}

Effectiveness

Multi-component intervention

Behavioral change

HIV/AIDS prevention

Young people

\section{Palabras clave}

Efectividad

Intervención multicomponente

Cambio conductual

Prevención VIH/Sida

Jóvenes

\section{Introduction}

Young people aged 15-24 years are approximately $20 \%$ of new HIV infections and $11 \%$ of HIV diagnoses in European Region in 2015 [1]. In Spain, the greater rate of HIV infection occurred before age $30(27.2 \%)$ according to the Spanish Information System of New HIV Diagnoses (SINIVIH) [2]. The average age of new HIV diagnoses was 36 years old, but $46.5 \%$ of them showed late diagnoses. Nowadays AIDS is a treatable and chronic disease, however it still remains incurable and break into the lives of young people significantly; it impacts on the lifestyle and expectations of their future, it interferes with self-image and self-esteem, and it alters their participation in social and work life [3]. 
Young people are vulnerable to HIV infection. They still have misconceptions and lack of knowledge about routes of HIV transmission and sexual risk practices, low perceived vulnerability, multiple sexual partners, alcohol and others drugs consumption and poor sexual assertiveness skills [4, 5, 6, 7]. Behavioral interventions reduce sexual risk behavior and prevent sexual transmitted infections (STI) $[8,9,10,11]$. The mainly outcomes of HIV preventive interventions among young people across different countries showed positive effects on knowledge, attitudes or behavioral intention $[12,13,14]$ and on condom use behavior $[10,15,16,17,18]$. However, most of the prevention interventions among young people in Europe reviewed by Lazarus, SihvonenRiemenschneider, Laukamm-Josten, Wong and Liljestrand had not a verified effectiveness, were not successful in changing sexual risk behavior or their changes decreased at long-term [19].

Most of the Spanish HIV prevention programs were school-based specially targeted to adolescents. They focused on providing information about HIV, STD or contraception and condom use attitudes trough: educational talks, out-of-class activities (e.g., health center visit, exhibition of posters, projection of documentary films) and social communication activities (e.g., design of advertising messages). Some of these included demonstrations of correct condom use or role-plays about scenarios related to HIV prevention. Few of them were current: e.g., PRESSEC [20], Sida: Saber Ayuda [21], AulaSida [22], Parlem Clar [23], Educational Intervention [24, 25], Parlem-ne; no et tallis [26]. A recent review by Espada, Morales, Orgilés, Piqueras and Carballo found no clear effectiveness assessment, a limited extrapolation of outcomes and studies typically focused on changes in attitudes and knowledge [27]. The program Skills for Adolescents with a Healthy Sexuality or COMPAS [28] was designed to improve these disadvantages in adolescents aged 15-18 years old. It was a cultural adaptation of ¡Cuídate!, specific HIV education program for Latino adolescents from the United States, that included five components: information, social skills, troubleshooting, maintenance strategies and selfinstructions and covert behavior rehearsal. In Spain only two studies were based on intervention effectiveness in young people: the first evaluated the differential effectiveness of six isolated techniques for preventing HIV/AIDS [29] and the second explored the effects of a sexual health information brochure in prevention of STIs [30]. Ballester-Arnal et al. [29] showed improvement of preventive factors through the application of intervention techniques comparing to non-intervention. Specially, the motivational techniques (attitudinal discussion or seropositive facilitator participation) revealed more effectiveness for protective behaviors. On the other hand, García-Retamero and Cokely [30] observed that gain-framed prevention messages are more effective to improve 
the positive attitudes towards condom use and to engage in protective behaviors; whereas loss-framed prevention messages were more effective to promote STD's screening behavior.

Thus, experimental studies that analyze the long-term impact of a brief HIV preventive interventions on key change sexual risk behavior components in Spanish young population have not been found. Nevertheless, evidence-based interventions may have significant effects on disease prevention and consequently may reduce personal burden and health care cost. In addition, brief interventions may reduce temporal, personal and economic costs and may increase the implementation feasibility in different settings. The purpose of this study was to assess the effectiveness of a brief intervention on knowledge, attitudes, beliefs and behaviors related to HIV/AIDS at short-medium-long term.

\section{Methods}

\section{Participants}

Eligibility criteria were based on the risk characteristics of HIV infection in our context. They included being Spanish and aging between 18 and 25 years old. An intentional non-probabilistic sampling plan was used and 467 young people participated at baseline. Additionally, participants were assessed a week $(\mathrm{n}=346)$, a month $(\mathrm{n}=324)$, three months $(\mathrm{n}=331)$ and a year $(\mathrm{n}=101)$ after providing the HIV preventive intervention.

Participants were on average twenty years old (58.7\% were women and $41.3 \%$ were men) (see Table 1). Most of them self-identified as heterosexual (91.2\%) and some of them as homosexual (5.6\%) or bisexual (3.2\%). However, $11.8 \%$ had had same-sex practices. They lived in urban areas and belonged to middle class. $70.4 \%$ had studied at high school and a $29.6 \%$ had got their degree. Concerning sexual experience, $89.9 \%$ of participants reported mutual masturbation, followed by vaginal sex $(88.8 \%)$, oral sex $(87.1 \%)$ and anal sex (30.5\%). They had had an average of five sexual partners and $63.8 \%$ had steady partner at the moment of evaluation.

\section{Table 1}

Sociodemographic characteristics over time

\begin{tabular}{|l|l|l|l|l|}
\hline Pre & Post & 1 month & 3 months & 1 year \\
\hline $\mathrm{N}=467$ & $\mathrm{~N}=346$ & $\mathrm{~N}=324$ & $\mathrm{~N}=331$ & $\mathrm{~N}=101$ \\
$(\mathbf{1 0 0 \% )}$ & $(\mathbf{7 4 . 1 \% )}$ & $(\mathbf{6 9 . 4 \% )}$ & $(\mathbf{7 0 . 9 \% )}$ & $(\mathbf{2 1 . 6 \% )}$ \\
\hline
\end{tabular}

Age 


\begin{tabular}{|c|c|c|c|c|c|}
\hline & Pre & Post & 1 month & 3 months & 1 year \\
\hline & $\begin{array}{l}N=467 \\
(100 \%)\end{array}$ & $\begin{array}{l}N=346 \\
(74.1 \%)\end{array}$ & $\begin{array}{l}N=324 \\
(69.4 \%)\end{array}$ & $\begin{array}{l}N=331 \\
(70.9 \%)\end{array}$ & $\begin{array}{l}N=101 \\
(21.6 \%)\end{array}$ \\
\hline $\begin{array}{l}\text { Mean } \\
\text { (SD) }\end{array}$ & $20.62(2.14)$ & $\begin{array}{l}20.61 \\
(2.012)\end{array}$ & $20.63(2.13)$ & $20.55(2.09)$ & $20.67(0.08)$ \\
\hline \multicolumn{6}{|l|}{ Gender } \\
\hline Male & $41.3 \%$ & $41 \%$ & $39.8 \%$ & $39 \%$ & $26.7 \%$ \\
\hline Female & $58.7 \%$ & $59 \%$ & $60.2 \%$ & $61 \%$ & $73.3 \%$ \\
\hline
\end{tabular}

\section{Procedure}

This study used a longitudinal design to assess the short, medium and long-term effectiveness of a Brief Program for AIDS Prevention in Young (B-PAPY). The overall goal was to reduce risk factors for HIV infection and sexual risk behaviors. Participants were recruited through face-to-face in Spanish college campuses in awareness-raising and information activities on World AIDS Day in December. More information about the experiment was provided by e-mail or phone. The procedure followed the ethical principles of A.P.A. The completion of questionnaires (about $1 \mathrm{~h}$ ) was conducted in the university facilities, individually and supervised by the staff of our Research Unit. The written consent of all participants was obtained. This phase lasted about two months. Then, we began the intervention design. The development of the intervention content was based on the key components of behavioral change theories, scientific literature on well-established programs and previous studies of our own Research Unit. Two sexual educators who had been previously trained in HIV prevention delivered program sessions. The B-PAPY had a small grouporiented framework (10-12 participants) and consisted of a workshop of a onesession $(3 \mathrm{~h})$. The addressed topics were:

(1) Information about routes of HIV transmission, risk and healthy practices (misconceptions), HIV antibody testing and treatment of AIDS (25 min).

(2) Perception of risk of HIV infection (20 min).

(3) Perceived competence to use condoms, attitudes towards condom use, behavioral skills (40 $\mathrm{min}$ ).

(4) Sexual assertiveness, self-management of safe decisions, emotional selfregulation (65 $\mathrm{min})$.

(5) Stigma, empathy towards people living with HIV/AIDS, perceived impact 
of living with AIDS (30 min).

Activities included games (e.g., cards), interactive exercises (e.g., finishing incomplete sentences), group discussion (e.g., defending the benefits vs. barriers to use of condoms, the role of pleasure, the fear of negative evaluation, etc.), role play (e.g., correctly putting and removing a condom, refusing a sexual risk intercourse, proposing use of a condom to a partner, etc.) and debate (e.g., real testimonials of people living with HIV).

Participants received compensation up to 20 euros for completing the 1-year follow up questionnaires. The procedure was approved by the Institutional Ethics Committee of the University.

\section{Evaluation}

AIDS Prevention Questionnaire (Cuestionario de Prevención del Sida or CPS) [31] is a self-administered instrument including 65 questions. They are divided into six components: information, risk perception, attitudes and beliefs, behavioral intention, risky behavior and solidarity towards people living with HIV. The internal consistency and test-retest reliability of the data were acceptable, obtaining a Cronbach's Alpha value of 0.70 and a correlation of 0.83 between test and retest. The outcome domains analyzed for intervention efficacy were:

(a) HIV/AIDS Knowledge. A series of 27 statements about routes of transmission, risky practices, myths and misconceptions. Dichotomous responses included "Yes" or "No". Scores ranged from 0 to 1 points. For example: People living with AIDS without symptoms, can transmit HIV?

(b) Risk Perception. 3-items that ask the perceived susceptibility ("Notes from 0 to 100: The likelihood or risk you perceive to get HIV"), fear of disease ("Notes from 0 to 100: what fear will produce the possibility to get HIV") and perceived severity (the responses were on a scale of "Low" to "Fatal").

(c) Attitudes towards condom use. A selection of the 15 mainly benefits and barriers of using condoms. Dichotomous responses included "Yes" or "No". Scores range from 0 to 1 points. Additionally, an item that assessed the confidence in condom was scored with "None" to "High". For example: When you use condom, you feel less sexual pleasure.

(d) Condom use self-efficacy. A series of 7 statements that described condomuse related skills during a different time in sexual behavior. How capable 
you would feel to use, buy, talk, put or refuse sexual intercourse on a seven-point scale from 0 ("Absolutely disagree") to 6 ("Totally agree"). For example: I would be comfortable talking about condom use with a partner before starting sexual intercourse.

(e) Condom use. Six questions measured the frequency of protected practices in vaginal intercourse, oral sex and anal intercourse, sex with a steady partner, sex with an occasional partner and sex under alcohol/drugs effects. Responses ranged from 0 ("Never") to 3 (“Always"). For example: How often have you used condoms under alcohol and drugs influence?

(f) Perceived behavioral competence. An item that evaluated the perceived competence to use condom from 0 to 100. For example: Do you know how to use condoms in sexual intercourse?

\section{Analysis of Data}

Descriptive statistics were performed (mean and standard deviation or percentages of frequency) to explore information about the different independent variables at pretest, posttest and follow-ups. Secondly, we evaluated long-term intervention impact between baseline and one-year follow-up. Repeatedmeasures analysis of variance (ANOVA) with 5 times (T1, baseline; T2, 1 week; T3, 1 month; T4, 3 months; and T5, 1 year) were used for analyzing continuous variables such as HIV knowledge, perceived susceptibility, fear and perceived benefits or barriers. Friedman test was done for evaluating categorical variables such as perceived severity, confidence on condom, condom use or self-efficacy skills. We used SPSS-19 for statistical analyses. Moreover, we analyzed the effect sizes (Cohen's d); meaningful effect sizes were estimated as small $(d=0.20)$, moderate $(d=50)$, and large $(d=80)$ [32]. Additionally, it was calculated through the HLS-Meta program by Huedo-Medina, Lally and Sagherian [33] in non-parametric variables.

\section{Results}

\section{HIV/AIDS Knowledge}

The baseline HIV knowledge was 0.61 (ranged 0-1) and after the intervention the HIV knowledge increased in a $9.8 \%$ at week. This increase remained stable over time. Differences were statistically significant in all times $(p=0.001)$ and got a moderate effect size along the follow-ups (see Table 2).

\section{Table 2}

Impact of B-PAPY on information about HIV, perception of risk and attitudes towards condc 


\begin{tabular}{|c|c|c|c|c|c|c|c|}
\hline & Pre & Post & 1 month & 3 months & 1 year & \multirow{2}{*}{$\begin{array}{l}\text { ANOVA } \\
(\mathrm{df}=4)\end{array}$} & d (co \\
\hline & $\begin{array}{l}N=467 \\
(100 \%)\end{array}$ & $\begin{array}{l}N=346 \\
(74.1 \%)\end{array}$ & $\begin{array}{l}N=324 \\
(69.4 \%)\end{array}$ & $\begin{array}{l}N=331 \\
(70.9 \%)\end{array}$ & $\begin{array}{l}N=101 \\
(21.6 \%)\end{array}$ & & \multirow{2}{*}{$\begin{array}{l}\text { Pre- } \\
\text { post }\end{array}$} \\
\hline & $\begin{array}{l}\text { Mean } \\
\text { (SD) }\end{array}$ & $\begin{array}{l}\text { Mean } \\
\text { (SD) }\end{array}$ & $\begin{array}{l}\text { Mean } \\
\text { (SD) }\end{array}$ & $\begin{array}{l}\text { Mean } \\
\text { (SD) }\end{array}$ & $\begin{array}{l}\text { Mean } \\
\text { (SD) }\end{array}$ & (p) & \\
\hline Knowledge & $\begin{array}{l}0.61 \\
(0.12)\end{array}$ & $\begin{array}{l}0.67 \\
(0.09)\end{array}$ & $\begin{array}{l}0.67 \\
(0.09)\end{array}$ & $\begin{array}{l}0.66 \\
(0.10)\end{array}$ & $\begin{array}{l}0.67 \\
(0.08)\end{array}$ & $\begin{array}{l}11.22 \\
(.001)\end{array}$ & $\begin{array}{l}0.498 \\
(\mathbf{0 . 3 8} \\
\mathbf{0 . 6 1 0}\end{array}$ \\
\hline $\begin{array}{l}\text { Perceived } \\
\text { susceptibility }\end{array}$ & $\begin{array}{l}17.66 \\
(22.72)\end{array}$ & $\begin{array}{l}20.93 \\
(23.86)\end{array}$ & $\begin{array}{l}22.22 \\
(24.63)\end{array}$ & $\begin{array}{l}20.91 \\
(23.92)\end{array}$ & $\begin{array}{l}22.21 \\
(23.63)\end{array}$ & $\begin{array}{l}2.98 \\
(.019)\end{array}$ & $\begin{array}{l}0.143 \\
(0.03 \\
0.249\end{array}$ \\
\hline Fear & $\begin{array}{l}69.72 \\
(36.10)\end{array}$ & $\begin{array}{l}75.75 \\
(31.27)\end{array}$ & $\begin{array}{l}76.43 \\
(30.55)\end{array}$ & $\begin{array}{l}75.63 \\
(31.61)\end{array}$ & $\begin{array}{l}76.41 \\
(32.98)\end{array}$ & $\begin{array}{l}2.02 \\
(.091)\end{array}$ & $\begin{array}{l}0.166 \\
(0.06 \\
0.272\end{array}$ \\
\hline $\begin{array}{l}\text { Perceived } \\
\text { benefits }\end{array}$ & $\begin{array}{l}0.39 \\
(0.15)\end{array}$ & $\begin{array}{l}0.42 \\
(0.14)\end{array}$ & $\begin{array}{l}0.41 \\
(0.15)\end{array}$ & $\begin{array}{l}0.41 \\
(0.14)\end{array}$ & $\begin{array}{l}0.42 \\
(0.22)\end{array}$ & $\begin{array}{l}1.52 \\
(.196)\end{array}$ & $\begin{array}{l}0.199 \\
0.09 \\
0.305\end{array}$ \\
\hline $\begin{array}{l}\text { Perceived } \\
\text { barriers }\end{array}$ & $\begin{array}{l}0.16 \\
(0.09)\end{array}$ & $\begin{array}{l}0.15 \\
(0.08)\end{array}$ & $\begin{array}{l}0.15 \\
(0.09)\end{array}$ & $\begin{array}{l}0.15 \\
(0.08)\end{array}$ & $\begin{array}{l}0.14 \\
(0.09)\end{array}$ & $\begin{array}{l}1.38 \\
(.240)\end{array}$ & $\begin{array}{l}-0.11 \\
(-0.2 \\
-0.06\end{array}$ \\
\hline $\begin{array}{l}\text { Perceived } \\
\text { competence }\end{array}$ & $\begin{array}{l}81.28 \\
(20.79)\end{array}$ & $\begin{array}{l}91.42 \\
(11.34)\end{array}$ & $\begin{array}{l}91.56 \\
(11.29)\end{array}$ & $\begin{array}{l}90.30 \\
(12.62)\end{array}$ & $\begin{array}{l}91.50 \\
(10.51)\end{array}$ & $\begin{array}{l}15.60 \\
(.001)\end{array}$ & $\begin{array}{l}0.486 \\
(\mathbf{0 . 3 7} \\
0.598\end{array}$ \\
\hline
\end{tabular}

\section{Risk Perception}

The mean of HIV susceptibility perception prior to the intervention was low (17.66\%). This percentage increased progressively at short $(20.93 \%)$, medium (20.91\%) and long-term (22.21\%). The change was statistically significant $(\mathrm{p}=0.019)$ (see Table 2). Moreover, fear towards HIV infection increased about six or seven points at pre-test $(69.72 \%)$ and follow-ups. However, results by ANOVA were not statistically significant $(\mathrm{p}=0.091)$ (see Table 2). Additionally, there were changes statistically significant in the perceived severity of AIDS $(p=0.038)$ that became more realistic. The percentage of participants who considered AIDS as a fatal disease decreased, and as a consequence, the percentage of those who considered it as high severity increased (see Table 3). 


\section{Table 3}

Impact of B-PAPY on perceived severity of AIDS and confidence in condom use

\begin{tabular}{|c|c|c|c|c|c|c|c|c|}
\hline \multicolumn{2}{|c|}{$\begin{array}{l}\text { Pre There is a } \\
\text { mistake in the size } \\
\text { of this column. PRE } \\
\text { and } N=467 \\
(100 \%) \text { should be } \\
\text { placed just above } \\
\text { the column } \\
\text { containing the } \\
\text { data: } 0.4,2.8,58 \text {, } \\
\text { etc. }\end{array}$} & Post & 1 month & 3 months & 1 year & $\begin{array}{l}\text { Fried } \\
(\mathrm{df}=4)\end{array}$ & \multicolumn{2}{|c|}{ d (confidenc } \\
\hline \multicolumn{2}{|c|}{$N=467(100 \%)$} & $\begin{array}{l}N=346 \\
(74.1 \%)\end{array}$ & $\begin{array}{l}N=324 \\
(69.4 \%)\end{array}$ & $\begin{array}{l}N=331 \\
(70.9 \%)\end{array}$ & $\begin{array}{l}N=101 \\
(21.6 \%)\end{array}$ & (p) & $\begin{array}{l}\text { Pre- } \\
\text { post }\end{array}$ & $\begin{array}{l}\text { Pr } \\
\text { fo } \\
\text { ur }\end{array}$ \\
\hline \multicolumn{9}{|c|}{ Perceived severity } \\
\hline Low & 0.4 & 0 & 0 & 0 & 0 & \multirow{4}{*}{$\begin{array}{l}10.15 \\
(.038)\end{array}$} & \multirow{4}{*}{$\begin{array}{l}0.010 \\
(-0.095 \\
0.115)\end{array}$} & \multirow{4}{*}{$\begin{array}{l}0 . \\
(- \\
0 .\end{array}$} \\
\hline Moderate & 2.8 & 5.5 & 3.4 & 5.1 & 7 & & & \\
\hline High & 58 & 70.7 & 74.9 & 72.2 & 73 & & & \\
\hline Fatal & 38.8 & 23.9 & 21.7 & 22.7 & 20 & & & \\
\hline \multicolumn{9}{|c|}{ Confidence in condom } \\
\hline None & 0.2 & 0.3 & 0.3 & 0 & 0 & \multirow{4}{*}{$\begin{array}{l}34.56 \\
(.001)\end{array}$} & \multirow{4}{*}{$\begin{array}{l}-0.001 \\
(-0.107 \\
0.103)\end{array}$} & \multirow{4}{*}{$\begin{array}{l}-C \\
(- \\
0 .\end{array}$} \\
\hline Low & 5.6 & 0.9 & 1.5 & 1.2 & 2 & & & \\
\hline Moderate & 33.7 & 25.9 & 24.2 & 24.2 & 26 & & & \\
\hline High & 60.5 & 73 & 74 & 74.6 & 72 & & & \\
\hline
\end{tabular}

\section{Attitudes Towards Condom Use}

Post-intervention scores indicate that perceived benefits in use of condoms were greater and perceived barriers were minors than pre-test scores. There was an increase of $7.7 \%$ in attributed advantages towards condom use and a decrease of $6.25 \%$ in attributed disadvantages towards condom use, changes were not statistically significant ( $\mathrm{p}=0.196$ and $\mathrm{p}=0.240$, consecutively) (see Table 2 ). Moreover, confidence in condom was statistically significant higher from pretest to post-test and follow-ups $(p=0.001)$ (see Table 3$)$.

\section{Condom Use Self-Efficacy}


Belief in own ability to use condoms increased slightly over time, but there were not statistically significant differences (see Table 2). However, there was one statistically significant difference between baseline scores and follow-ups in feeling confident about buying condoms $(\mathrm{p}=0.009)$ (see Table 4). In addition, perceived competence to use correctly condom also increased. This outcome got a moderate effect size along the follow-ups.

\section{Table 4}

Impact of B-PAPY on condom use self-efficacy skills

\begin{tabular}{|c|c|c|c|c|c|c|c|}
\hline \multicolumn{2}{|c|}{$\begin{array}{l}\text { Pre There is a mistake } \\
\text { in the size of this column. } \\
\text { PRE, } N=467(100 \%) \text { and } \\
\text { Mean (SD) should be } \\
\text { placed just above the } \\
\text { column containing the } \\
\text { data: } 4.88,4.96,5.21 \text {, } \\
\text { etc. }\end{array}$} & Post & 1 month & 3 months & 1 year & $\begin{array}{l}\text { ANOVA } \\
(d f=4)\end{array}$ & d (con: \\
\hline \multicolumn{2}{|c|}{$\mathrm{N}=467(100 \%)$} & $\begin{array}{l}N=346 \\
(74.1 \%)\end{array}$ & $\begin{array}{l}N=324 \\
(69.4 \%)\end{array}$ & $\begin{array}{l}N=331 \\
(70.9 \%)\end{array}$ & $\begin{array}{l}N=101 \\
(21.6 \%)\end{array}$ & (p) & \multirow{2}{*}{$\begin{array}{l}\text { Pre- } \\
\text { post }\end{array}$} \\
\hline \multicolumn{2}{|l|}{ Mean (SD) } & $\begin{array}{l}\text { Mean } \\
\text { (SD) }\end{array}$ & $\begin{array}{l}\text { Mean } \\
\text { (SD) }\end{array}$ & $\begin{array}{l}\text { Mean } \\
\text { (SD) }\end{array}$ & $\begin{array}{l}\text { Mean } \\
\text { (SD) }\end{array}$ & & \\
\hline $\begin{array}{l}\text { Buying } \\
\text { condoms }\end{array}$ & $\begin{array}{l}4.88 \\
(1.27)\end{array}$ & $\begin{array}{l}4.97 \\
(1.28)\end{array}$ & $\begin{array}{l}5.01 \\
(1.29)\end{array}$ & $\begin{array}{l}5.10 \\
(1.25)\end{array}$ & $\begin{array}{l}4.99 \\
(1.30)\end{array}$ & $\begin{array}{l}2.1 \\
(.077)\end{array}$ & $\begin{array}{l}0.070 \\
(-0.03 \\
0.176)\end{array}$ \\
\hline $\begin{array}{l}\text { Talking about } \\
\text { condom use }\end{array}$ & $\begin{array}{l}4.96 \\
(1.14)\end{array}$ & $\begin{array}{l}5.07 \\
(1.11)\end{array}$ & $\begin{array}{l}5.15 \\
(1.06)\end{array}$ & $\begin{array}{l}5.0 \\
(1.06)\end{array}$ & $\begin{array}{l}4.99 \\
(1.45)\end{array}$ & $\begin{array}{l}0.96 \\
(.428)\end{array}$ & $\begin{array}{l}0.096 \\
(-0.00 \\
0.201)\end{array}$ \\
\hline $\begin{array}{l}\text { Fear of } \\
\text { rejection* }\end{array}$ & $\begin{array}{l}5.21 \\
(1.06)\end{array}$ & $\begin{array}{l}5.27 \\
(0.99)\end{array}$ & $\begin{array}{l}5.31 \\
(0.94)\end{array}$ & $\begin{array}{l}5.37 \\
(0.92)\end{array}$ & $\begin{array}{l}5.39 \\
(0.82)\end{array}$ & $\begin{array}{l}0.67 \\
(.611)\end{array}$ & $\begin{array}{l}0.056 \\
(-0.04 \\
0.161)\end{array}$ \\
\hline $\begin{array}{l}\text { Fear of } \\
\text { negative } \\
\text { evaluation* }\end{array}$ & $\begin{array}{l}5.29 \\
(1.06)\end{array}$ & $\begin{array}{l}5.35 \\
(0.98)\end{array}$ & $\begin{array}{l}5.35 \\
(1.02)\end{array}$ & $\begin{array}{l}5.28 \\
(1.11)\end{array}$ & $\begin{array}{l}5.19 \\
(1.28)\end{array}$ & $\begin{array}{l}1.00 \\
(.408)\end{array}$ & $\begin{array}{l}0.056 \\
(-0.04 \\
0.161)\end{array}$ \\
\hline $\begin{array}{l}\text { Remembering } \\
\text { use under } \\
\text { alcohol/drugs } \\
\text { influence }\end{array}$ & $\begin{array}{l}4.81 \\
(1.22)\end{array}$ & $\begin{array}{l}4.75 \\
(1.19)\end{array}$ & $\begin{array}{l}4.79 \\
(1.10)\end{array}$ & $\begin{array}{l}4.79 \\
(1.16)\end{array}$ & $\begin{array}{l}4.77 \\
(1.32)\end{array}$ & $\begin{array}{l}0.89 \\
(.469)\end{array}$ & $\begin{array}{l}-0.049 \\
(-0.15 \\
0.056)\end{array}$ \\
\hline $\begin{array}{l}\text { Putting on a } \\
\text { condom }\end{array}$ & $\begin{array}{l}4.76 \\
(1.49)\end{array}$ & $\begin{array}{l}4.80 \\
(1.47)\end{array}$ & $\begin{array}{l}4.71 \\
(1.54)\end{array}$ & $\begin{array}{l}4.60 \\
(1.64)\end{array}$ & $\begin{array}{l}4.78 \\
(1.61)\end{array}$ & $\begin{array}{l}1.86 \\
(.116)\end{array}$ & $\begin{array}{l}0.026 \\
(-0.07 \\
0.132)\end{array}$ \\
\hline $\begin{array}{l}\text { Stopping } \\
\text { maximum } \\
\text { excitation }\end{array}$ & $\begin{array}{l}4.13 \\
(1.58)\end{array}$ & $\begin{array}{l}4.34 \\
(1.48)\end{array}$ & $\begin{array}{l}4.45 \\
(1.39)\end{array}$ & $\begin{array}{l}4.37 \\
(1.46)\end{array}$ & $\begin{array}{l}4.57 \\
(1.39)\end{array}$ & $\begin{array}{l}0.35 \\
(.841)\end{array}$ & $\begin{array}{l}0.132 \\
(0.026 ; \\
0.238)\end{array}$ \\
\hline
\end{tabular}


Pre There is a mistake in the size of this column. PRE, $N=467(100 \%)$ and Mean (SD) should be placed just above the column containing the data: $4.88,4.96,5.21$, etc.

\begin{tabular}{|c|c|c|c|c|c|c|c|}
\hline \multicolumn{2}{|c|}{$N=467(100 \%)$} & $\begin{array}{l}N=346 \\
(74.1 \%)\end{array}$ & $\begin{array}{l}N=324 \\
(69.4 \%)\end{array}$ & $\begin{array}{l}N=331 \\
(70.9 \%)\end{array}$ & $\begin{array}{l}N=101 \\
(21.6 \%)\end{array}$ & (p) & \multirow{2}{*}{$\begin{array}{l}\text { Pre- } \\
\text { post }\end{array}$} \\
\hline \multicolumn{2}{|l|}{ Mean (SD) } & $\begin{array}{l}\text { Mean } \\
\text { (SD) }\end{array}$ & $\begin{array}{l}\text { Mean } \\
\text { (SD) }\end{array}$ & $\begin{array}{l}\text { Mean } \\
\text { (SD) }\end{array}$ & $\begin{array}{l}\text { Mean } \\
\text { (SD) }\end{array}$ & & \\
\hline Self-efficacy & $\begin{array}{l}34.07 \\
(4.87)\end{array}$ & $\begin{array}{l}34.56 \\
(4.46)\end{array}$ & $\begin{array}{l}34.79 \\
(4.67)\end{array}$ & $\begin{array}{l}34.63 \\
(4.61)\end{array}$ & $\begin{array}{l}34.67 \\
(5.12)\end{array}$ & $\begin{array}{l}0.32 \\
(.859)\end{array}$ & $\begin{array}{l}0.100 \\
(-0.00 \\
0.206)\end{array}$ \\
\hline
\end{tabular}

\section{Protective Behaviors}

There was an increase statistically significant in the systematic use of condoms in vaginal intercourse $(p=0.002)$ and sex in a steady partner $(p=0.040)$. In both cases, this increase remained stable at short-term and medium-term, but took the initial values at one year evaluation. Protective sexual behaviors also increased in the other sex practices and situations, but there were not statistically significant differences (see Table 5).

\section{Table 5}

Impact of B-PAPY on protective behaviors in different sex situations

\section{Pre There is a} mistake in the size of this column. PRE and $\mathrm{N}=467(100 \%)$ should be placed just above the column containing the data: $1.5,16.4,42.8$, etc.
Post 1 month 3 months
1 year

Fried $(\mathbf{d f}=4)$ d (confide

Prepost

Vaginal

\begin{tabular}{|l|l|l|l|l|l|l|l|}
\hline Never & 1.5 & 25 & 26.2 & 23.4 & 17.9 & $\mathbf{1 7 . 1 8}$ & $\begin{array}{l}-0.004 \\
(-0.109\end{array}$ \\
\hline Sometimes & 16.4 & 9.1 & 9.6 & 10.9 & 20.2 & & $0.102)$
\end{tabular}


Pre There is a mistake in the size of this column. PRE and $\mathrm{N}=467(100 \%)$

should be placed just above the column containing the data: $1.5,16.4,42.8$, etc.

\section{Post}

\begin{tabular}{l|l|l}
1 month & 3 months 1 year
\end{tabular}
Fried $(\mathrm{df}=4)$ d (confide

(p) Pre-
post

\begin{tabular}{|l|l|l|l|l|l|}
$\mathbf{N}=\mathbf{4 6 7}(\mathbf{1 0 0 \% )}$ & $\mathbf{( 7 4 . 1 \% )}$ & $\mathbf{( 6 9 . 4 \% )}$ & $\mathbf{( 7 0 . 9 \% )}$ & $\mathbf{( 2 1 . 6 \%})$ \\
\hline Often & 42.8 & 13.8 & 16.6 & 18.5 & 22.6 \\
\hline Always & 39.4 & 52.2 & 47.6 & 47.2 & 39.3 \\
\hline
\end{tabular}

Oral

\begin{tabular}{|c|c|c|c|c|c|c|c|}
\hline Never & 63.5 & 77 & 78.2 & 78.5 & 80.2 & \multirow{4}{*}{$\begin{array}{l}3.84 \\
(.427)\end{array}$} & \multirow{3}{*}{$\begin{array}{l}-0.087 \\
(-0.193 \\
0.018)\end{array}$} \\
\hline Sometimes & 24 & 11.7 & 9.7 & 113.1 & 8.6 & & \\
\hline Often & 8.6 & 4.1 & 4.9 & 3.1 & 3.7 & & \\
\hline Always & 3.9 & 7.1 & 7.3 & 7.5 & 7.4 & & \\
\hline
\end{tabular}

Anal

\begin{tabular}{|l|l|l|l|l|l|l|l|}
\hline Never & 34.8 & 45.1 & 58.3 & 56 & 60.9 & & \\
\hline Sometimes & 21.3 & 7.8 & 6.3 & 8 & 8.7 & 4.00 & $\begin{array}{l}-0.005 \\
(-0.110 ;\end{array}$ \\
\hline Often & 15.9 & 11.8 & 8.3 & 6 & 0 & $(.406)$ & $0.101)$ \\
\hline Always & 28 & 35.3 & 27.1 & 30 & 30.4 & & \\
\hline
\end{tabular}

Steady partner

\begin{tabular}{|c|c|c|c|c|c|c|c|}
\hline Never & 9.7 & 25.2 & 29.8 & 31.9 & 27.8 & \multirow{4}{*}{$\begin{array}{l}10.02 \\
(.040)\end{array}$} & \multirow{4}{*}{$\begin{array}{l}-0.005 \\
(-0.111 \\
0.100)\end{array}$} \\
\hline Sometimes & 24.8 & 12.1 & 10.6 & 13.1 & 22.2 & & \\
\hline Often & 34.1 & 19.4 & 15.7 & 18.8 & 19.4 & & \\
\hline Always & 31.4 & 43.2 & 43.9 & 36.2 & 30.6 & & \\
\hline
\end{tabular}

Occasional partner

\begin{tabular}{|l|l|l|l|l|l|l|l|}
\hline Never & 8.3 & 19.8 & 16.7 & 17 & 14.3 & & \\
\hline Sometimes & 9.5 & 4.4 & 9.5 & 10.6 & 11.4 & 2.62 & $\begin{array}{l}-0.000 \\
(-0.106 ; \\
0.105)\end{array}$ \\
\hline Often & 19.8 & 11 & 16.7 & 16 & 17.1 & $(.623)$ & \\
\hline Always & 62.4 & 64.8 & 57.1 & 56.4 & 57.1 & & \\
\hline
\end{tabular}

Under alcohol/drugs influence

\begin{tabular}{l|l|l|l|l|l|l|l|} 
Never & 11 & 23.8 & 26.8 & 21.2 & 21.1 & 2.48 & -0.000
\end{tabular}




\begin{tabular}{|c|c|c|c|c|c|c|c|}
\hline \multicolumn{2}{|c|}{$\begin{array}{l}\text { Pre There is a } \\
\text { mistake in the size of } \\
\text { this column. PRE and } \\
N=467(100 \%) \\
\text { should be placed just } \\
\text { above the column } \\
\text { containing the data: } \\
1.5,16.4,42.8 \text {, etc. }\end{array}$} & Post & 1 month & 3 months & 1 year & $\begin{array}{l}\text { Fried } \\
(\mathrm{df}=4)\end{array}$ & $\begin{array}{l}(-0.106 \\
0.105)\end{array}$ \\
\hline \multicolumn{2}{|c|}{$N=467(100 \%)$} & $\begin{array}{l}N=346 \\
(74.1 \%)\end{array}$ & $\begin{array}{l}N=324 \\
(69.4 \%)\end{array}$ & $\begin{array}{l}N=331 \\
(70.9 \%)\end{array}$ & $\begin{array}{l}N=101 \\
(21.6 \%)\end{array}$ & (p) & $\begin{array}{l}\text { Pre- } \\
\text { post }\end{array}$ \\
\hline Sometimes & 18.4 & 13.9 & 16.1 & 15.3 & 17.5 & & \\
\hline Often & 25.5 & 16.6 & 13.1 & 17.6 & 15.8 & & \\
\hline Always & 45.2 & 45.7 & 44 & 45.9 & 45.6 & & \\
\hline
\end{tabular}

\section{Discussion}

According to literature, this study is the first in the Spanish context aimed to evaluate the effectiveness of a single-session multi-component intervention on risky sexual behavior and other related factors among young people. Our outcomes have shown positive effects post-intervention in almost all evaluated variables. Young people had significant improvement in knowledge about HIV/AIDS, perceived HIV susceptibility, perceived competence, realistic perceived severity of AIDS or confidence in condom. These results are consistent with those obtained in other HIV interventions [12, 14, 28]. Certainly, truthful and objective information, good risk analysis and perceived positive benefits towards protection measures are necessary elements for effective HIV prevention. Moreover, a more realistic view of the seriousness of AIDS will improve the social perception of people living with HIV and secondary prevention actions such as HIV antibody testing [34]. Furthermore, our results have shown a clinical increase in self-efficacy skills and perceived benefits as well as a decrease in perceived barriers, but they were statistically nonsignificant. Perhaps, it would be necessary to increase time in motivational messages, or activities addressed to emotion-focused coping [29, 30].

With regard to the most important target behavior for HIV prevention, a greater condom use was observed in the follow-ups in line with previous studies in Spanish adolescents [24], American college students [16] or African American urban youth [18]. In our sample this increase was significant in vaginal sex and in steady sexual partner. These results are relevant because vaginal intercourse 
and serial monogamy are the riskier situations in this group. The work about real and close experiences to young people have a positive influence on the behavioral change. As Ballester-Arnal, Gil-Llario, Giménez-García and Kalichman found, the use of motivational techniques is more effective to get protective behaviors [29]. The systematic use of condoms in oral and anal sex, and sex under the effects of alcohol and drugs increased clinically but was not statistically significant. It is possible that condom negotiation in these sexual situations become more difficult due to the contextual characteristics of these sexual practices [35].

Finally, the change is maintained over time in knowledge, attitudes and beliefs. However, the long-term efficacy decrease at year in one behavioral variable. Adopting and maintaining safe sex behaviors with a regular partner become difficult because its particular affective characteristics and beliefs [36]. The interpersonal behavior is influenced by the dynamics of individuals involved in the relationship. Sex without condom o using another birth control method could appear due to aspects such as non-spontaneity, lack of love or confidence, associated with the use of condoms [37]. From the Change Transtheoretical Model, a one-session program would not be sufficient to maintain a protective behavior to long-term (action vs. maintenance process of change). Therefore, intervention may be improved with the application of the innovative and brief remembering strategies trough information and communications technology (ICTs) (e.g., helping relationships, counter-conditioning, reinforcement management, and stimulus control) to help to achieve behavior change maintenance [38]. Moreover, it could be interesting to include ICTs in the follow-up assessments for motivating their fulfillment and expanding the assessment time.

AQ1

Several limitations may be addressed in the future. A high percentage of participants were lost at the follow-up year, which may influence the magnitude of the statistical significance at long-term. Secondly, lack of balance in some variables such as sexual orientation, type of partner or gender has not allowed the differential analysis of effectiveness. Third, it would be important to deepen the knowledge of the contextual factors that involve sexual relationships under the effects of drugs (for example: type of drug consumed, frequency of consumption, motivations of consumption, perceived benefits, etc.). Finally, young people with higher education are representative of the Spanish population, moreover, the scientific literature has shown that high academic degree is not related to more preventive information or safe sex behaviors. However, the generalization of these results to other specific high-risk groups (such as MSM, MSW, etc.) should be viewed with caution. 
The practical implication of this study is that a multi-component one-session prevention program based on behavioral change theories increase the use of condom and reduce the risk of HIV infection in Spanish young people. This design has temporal, economic and personal advantages, thus they can fit into existing public health services. These results highlights the sexual health programs as a useful resource for HIV, STI and unwanted pregnancies, as well as a tool that should be part of the public health policies.

\section{Acknowledgements}

This work was supported by Universidad Jaume I-Fundación Bancaixa (P1 1B2006-19) and by the Spanish Foundation for AIDS Research and Prevention known as Fundación para la Investigación y la Prevención del Sida en España (exp. 36639/07).

Compliance with Ethical Standards

Conflict of interest The authors declare no competing interests.

Ethical Approval All procedures performed in studies involving human participants were in accordance with the ethical standards of the institutional and/or national research committee and with the 1964 Helsinki declaration and its later amendments or comparable ethical standards.

Informed Consent Informed consent was obtained from all individual participants included in the study.

\section{References}

1. European Centre for Disease Prevention and Control. WHO Regional Office for Europe. HIV/AIDS surveillance in Europe 2015. Stockholm: ECDC; 2016.

2. Subdirectorate-General for Health Promotion and Epidemiology. Epidemiological surveillance of HIV and AIDS in Spain: information system on new HIV diagnoses and National Registry of AIDS Cases. Madrid: ISCIII; 2016.

3. Iglesias I, González JC, León JA. Impacto del VIH en adolescentes y jóvenes: análisis preliminar desde la Teoría Cultural del Consenso. Rev Multidiscip Sida. 2015;1(4):40-50. 
4. Ballester R, Gil MD, Giménez C, Ruiz E. Actitudes y conductas sexuales de riesgo para la infección por VIH/Sida en jóvenes españoles. Rev Psicopatol Psicol Clín. 2009;14(3):181-91.

5. Ballester R, Gil MD, Ruiz E, Giménez C. Autoeficacia en la prevención sexual del Sida: la influencia del género. Anales de Psicología. 2013;29(1):76-82.

6. Díez M, Oliva J, Sánchez F, Vives N, Cervalló C, Izquierdo A. Incidence of new HIV diagnoses in Spain, 2004-2009. Gac Sanit. 2012;26:107-15.

7. Folch C, Álvarez JL, Casabona J, Brotons M, Castellsagué X, Grupo Jóvenes e Internet. Determinantes de las conductas sexuales de riesgo de jóvenes en Cataluña. Rev Esp Salud Pública. 2015; 89: 471-85.

8. Althoff MD, Grayson CT, Witt L, Holden J, Reid D, Kissinger P. A metaanalysis of the efficacy of behavioral interventions to reduce risky sexual behavior and decrease sexually transmitted infections in Latinas living in the United States. Health Educ Behav. 2015;42(6):709-18.

9. Eaton LA, Huedo-Medina TB, Kalichman SC, et al. Meta-analysis of single-session behavioral interventions to prevent sexually transmitted infections: implications for bundling prevention packages. Am J Public Health. 2012;102(11):e34-44.

10. Kennedy SB, Nolen S, Pan Z, Smith B, Applewhite J, Vanderhoff KJ. Effectiveness of a brief condom promotion program in reducing risky sexual behaviours among African American men. J Eval Clin Pract. 2013;19(2):40813.

11. Scott-Sheldon LA, Huedo-Medina TB, Warren MR, Johnson BT, Carey MP. Efficacy of behavioral interventions to increase condom use and reduce sexually transmitted infections: a meta-analysis, 1991-2010. J Acquir Immune Defic Syndr. 2011;58(5):489-98.

12. Evans AE, Edmundson-Drane EW, Harris KK. Computer-assisted instruction: an effective instructional method for HIV prevention education? J Adolesc Health. 2000;26(4):244-51.

13. Kaufman CE, Whitesell NR, Keane EM, et al. Effectiveness of Circle of Life, an HIV-preventive intervention for American Indian middle school 
youths: a group randomized trial in a Northern Plains tribe. Am J Public Health. 2014;104(6):e106-12.

14. Kudo Y. Effectiveness of a condom use educational program developed on the basis of the Information-Motivation-Behavioral Skills model. Jpn J Nurs Sci. 2013;10(1):24-40.

15. Mantell JE, Smit JA, Exner TM, et al. Promoting female condom use among female university students in KwaZulu-Natal, South Africa: results of a randomized behavioral trial. AIDS Behav. 2015;19(7):1129-40.

16. Norton WE, Fisher JD, Amico KR, Dovidio JF, Johnson BT. Relative efficacy of a pregnancy, sexually transmitted infection, or human immunodeficiency virus prevention-focused intervention on changing sexual risk behavior among young adults. J Am Coll Health. 2012;60(8):574-82.

17. Townsend L, Mathews C, Zembe Y. A systematic review of behavioral interventions to prevent HIV infection and transmission among heterosexual, adult men in low-and middle-income countries. Prev Sci. 2013;14(1):88-105.

18. Zellner T, Trotter J, Lenoir S, et al. Color it real: A program to increase condom use and reduce substance abuse and perceived stress. Int J Environ Res Public Health. 2015;13(1):51.

19. Lazarus JV, Sihvonen-Riemenschneider H, Laukamm-Josten U, Wong F, Liljestrand J. Systematic review of interventions to prevent spread of sexually transmitted infections, including HIV, among young people in Europe. Croat Med J. 2010;51(1):74-84.

20. Juárez O, Díez E. "PRESSEC". Prevenció de la sida des de l'escola secundaria. Barcelona: Publicació de l'Àrea de Salut Pública de l'Ajuntament de Barcelona; 1995.

21. Prats J. Un programa interdisciplinar para la Educación Secundaria: sida, saber ayuda. Cuadernos de Pedagogía. 1995;240:55-9.

22. Fernández C, Molina R, Ramírez C, et al. Cambios en las actitudes y conocimientos de los adolescentes sobre la infección por VIH tras la intervención escolar AULASIDA, 1996-1997. Rev Esp Salud Pública. 2000;74:163-76. 
23. Juárez O, Díez, E. "PARLEM CLAR". Prevenció de la sida a l'escola secundaria post-obligatoria. Barcelona: Publicacions de l'Institut Municipal de Salut Pública; 2002.

24. Callejas S, Fernández B, Méndez P, et al. Intervención educativa para la prevención de embarazos no deseados y enfermedades de transmisión sexual en adolescentes de la ciudad de Toledo. Rev Esp Salud Pública. 2005;79:5819 .

25. Rebull J, Reverté M, Piñas I, Ortí A, González LL, Contreras E. Evaluación pre-post de una actividad preventiva de la infección por VIH dirigida a los adolescentes de las comarcas del sur de Tarragona. Rev Esp Salud Pública. 2003;77:373-82.

26. Juárez O, Díez E. Parlem-ne; no et tallis! Programa escolar de salut sexual i comunicació. Barcelona: Agència de Salut Pública de Barcelona; 2010.

27. Espada JP, Morales A, Orgilés M, Piqueras JA, Carballo JL. A review of HIV/AIDS prevention programs in Spain. Int J Hisp Psychol. 2012;5:2.

28. Espada JP, Orgilés M, Morales A, Ballester R, Huedo-Medina TB. Effectiveness of a school HIV/AIDS prevention program for Spanish adolescents. AIDS Educ Prev. 2012;24(6):500-13.

29. Ballester-Arnal R, Gil-Llario MD, Giménez-García C, Kalichman SC. What works well in HIV prevention among Spanish young people? An analysis of differential effectiveness among six intervention techniques. AIDS Behav. 2015;19:1157-69.

30. García-Retamero R, Cokely ET. Effective communication of risks to young adults: using message framing and visual aids to increase Condom Use and STD Screening. J Expr Psychol. 2011;17(3):270-87.

31. Ballester R, Gil MD, Giménez C. El Cuestionario de Prevención del Sida (CPS): Análisis de la fiabilidad y validez. In Sida, un nuevo escenario: Proceedings of the X Congreso Nacional sobre el Sida de la Sociedad Española Interdisciplinaria de Sida. San Sebastián; Seisida; 2007. p. 135.

32. Cohen J. Statistical power analysis for the behavioral sciences. 2nd ed. Hillsdale: Lawrence Earlbaum Associates; 1988. 
33. Huedo-Medina TB, Lally N, Sagherian, M. HLS-meta: effect size converter to create an evidence-based database. Version 0.9, Storrs, CT; 2013.

34. Deblonde J, De Koker P, Hamers FF, Fontaine J, Luchters S, Temmerman M. Barriers to HIV testing in Europe: a systematic review. Eur J Public Health. 2010;20(4):422-32.

35. Moure-Rodríguez L, Doallo S, Juan-Salvadores P, Corral M, Cadaveira F, Caamaño-Isorna F. Consumo intensivo de alcohol y cannabis, y prácticas sexuales de riesgo en estudiantes universitarios. Gac Sanit. 2016;30(6):43843.

36. Macaluso M, Demand MJ, Artz LM, Hook EW 3rd. Partner type and condom use. AIDS. 2000;14(5):537-46.

37. VanderDrift LE, Agnew CR, Harvey SM, Warren JT. Whose intentions predict? Power over condom use within heterosexual dyads. Health Psychol. 2013;32(10):1038-46.

38. Haluza D, Jungwirth D. ICT and the future of health care: aspects of health promotion. Int J Med Inform. 2014;84(1):48-57. 